\title{
Soxhlet Extraction and Gas Chromatography Mass Spectrometry Analysis of Extracted Oil from Pistacia Atlantica Kurdica Nuts and Optimization of Process Using Factorial Design of Experiments
}

\author{
Atena Ghaderi, Bahram Ebrahimi* \\ Department of Chemistry, Faculty of Science, Sanandaj Branch, Islamic Azad University, Sanandaj, Iran \\ Email address: \\ B_ebrahimi@iausdj.ac.ir (B. Ebrahimi)
}

\section{To cite this article:}

Atena Ghaderi, Bahram Ebrahimi. Soxhlet Extraction and Gas Chromatography Mass Spectrometry Analysis of Extracted Oil from Pistacia Atlantica Kurdica Nuts and Optimization of Process Using Factorial Design of Experiments. Science Journal of Analytical Chemistry. Vol. 3, No. 6, 2015, pp. 122-126. doi: 10.11648/j.sjac.20150306.17

\begin{abstract}
Chemical constituents of extracted oil from the nut of Pistacia atlantica kurdica (dried and fresh samples) - which grows in some regions of Kurdistan province in Iran - were extracted by using soxhlet method and analyzed by gas chromatography mass spectrometry. The main goal of this study was qualitative analysis of extracted compounds and optimization of extraction conditions. At three extraction times (7h, 11h and 13h) and with two solvents (n-hexane and methanol), soxhlet extractions were carried out. Extraction time and type of solvents were selected as two important factors. A general full factorial design with selected factors was used. The study reveals that n-hexane extracted more yield than methanol. The highest yield obtained with n-hexane, was 33.59\%; while the highest yield obtained by methanol was $3.85 \%$. The results show that when extraction is done by n-hexane, by increasing the extraction time up to $11 \mathrm{~h}$, the mass yield percent was also increased. The obtained chromatograms were showed that by increasing the extraction time up to $13 \mathrm{~h}$, the main extracted compounds were maintained but compounds with low quantities have been eliminated. The main compounds identified were alpha-pinene, decane, dodecane, tetradecane, hexadecane, palmitic acid, oleic acid and linoleic acid ester. The main identified compounds were nearly similar to dried and fresh samples. This study can be considered as the firs report on the chemical compounds of extracted oil from nut of Pistacia atlantica kurdica in studied region.
\end{abstract}

Keywords: Pistacia Atlantica Kurdica, Soxhlet, Oil Extraction

\section{Introduction}

The use of natural herbal oils and resinous sap has been common throughout human life. These herbal products have been used in various fields especially in medicine use. Extraction and identification of chemical constituents of these products from various natural sources can be appealing for scientists. Indeed, in some cases we will encounter active plant ingredients that are novel or useful especially with medical specific effects. Morphine, quinine, camphor and cocaine are some of these examples $[1,2]$. With respect to side effects of synthetic drugs and other problems, a great change in mentality of manufacturers and consumers is happening gradually and herbal medicines are taken into consideration.

Anacardiaceae is a family of flowering plants in the order
Sapindales, with about 70 genera and 650 species of evergreen or deciduous trees, shrubs, and woody vines. Among this family, Anacardium occidentale and Pistaciavera are mostly well- known, the trees that are cultivated for their nuts (Cashew and Pistachio) [3]. Pistacia Atlantica Kurdica (p. a. Kurdica) is one of the subspecies of Pistacia atlantica. P. a. kurdica is spread around the Zagros Mountains and particularly in north-western of Iran, that is, especially in the Kurdish regions of Iran in Kurdistan Province. The extracted oils of some of the species of the genus Pistacia have been characterized [4-7]. Extraction from solid matrixes is very important in laboratory and industrial scale. Supercritical fluid extraction, pressurized fluid extraction and microwaveassisted extraction are well known modern methods in this field [8]. Essential oil extraction from various herbs is one of the important examples of extraction from solids. Soxhlet is 
one of the traditional methods based on solvent extraction that is applicable in these extractions. Optimized design of soxhlet extraction device has some advantages and decreases consuming of organic solvent. Sample is repeatedly brought into contact with the fresh parts of the solvent; the temperature of the system remains relatively high because the heat applied to the distillation flask reaches the extraction cavity to some extent, and no filtration is required after the leaching step.

Kurdistan, as one of the regions with herbal abundant diversity in the world [9], seems to be an excellent place for these studies. In this study the oil of fresh and dried nut samples from subspecies of P. a. kurdica, -which grows in some regions of Kurdistan province in Iran- were extracted using soxhlet method and analyzed by GC-MS. For optimization of extraction process a general full factorial design with two factors was implemented. The effects of selected factors (extractor solvent and time of extraction) were investigated and the yield of extraction was measured at different conditions. The extracted oil was analyzed by GCMS and Chemical constituents were identified. This study can be considered as the first report on the chemical compound of extracted oil from nut of P. a. kurdica in some regions of Kurdistan Province in Iran.

\section{Material and Methods}

\subsection{Chemicals}

All chemicals and reagents had analytical grade. Methanol and $n$-hexane with high purity were purchased from Dr. Mojallali industrial chemical complex Co. Tehran, Iran.

\subsection{Apparatus and Instruments}

The conventional Soxhlet extraction apparatus was used, which consists of a condenser, a Soxhlet chamber, and an extraction flask. The extractor thimble was permeable one with $44 \mathrm{~mm}$ internal diameter and $200 \mathrm{~mm}$ external length. The rotary evaporator was used for evaporation of solvent of extracted oil.

\subsection{Sampling}

The fresh sample of nut from P. a. Kurdica. tree was collected at the end of first month of summer in Kurdistan Province. In preparation of dried samples, the aliquots of fresh samples were spread in clean PTFE surface in sun light for one week. The samples were ground in grinding mill with particle size of less than $2 \mathrm{~mm}$. The raw grinded samples was sealed and stored in refrigerator for further usage.

\subsection{Percentage Yield of Extraction}

At the three selected times of extraction (7, 11 and $13 \mathrm{~h}$ ), twenty grams of prepared P.a. Kurdika samples were placed in thimble of Soxhlet apparatus and extracted with $350 \mathrm{ml}$ of an appropriate solvent for 7 hours, 11 hours and 13 hours. At any time, three extractions implemented with n-hexane and methanol as extractor solvents. The yields of extractions ( $\mathrm{Y}$ extract) were calculated by equation (2-1), where $\mathrm{X}$ extrac, is the crude extract mass $(\mathrm{g})$ and $\mathrm{X}$ herb is the extracted herb mass (g). All experiments with Soxhlet were triplicate for statistical evaluation.

$$
\mathrm{Y}_{\text {extract }}=\mathrm{X}_{\text {extract }} / \mathrm{X}_{\text {herb }} \times 100
$$

\subsection{Extracted oil Analysis (Gas Chromatography-Mass Spectrometry)}

An Agilent 6890 series Gas Chromatography and 5973 Mass Selective Detector was used. Samples were injected into HP-5 crosslinked 5\% phenyl methyl siloxane fusedsilica capillary column $(60 \mathrm{~m} \times 0.25 \mathrm{~mm}$ i.d., $\times$ film thickness $0.1 \mu \mathrm{m})$ in the splitless injection mode. The oven temperatures were as follows: the initial temperature was $50^{\circ} \mathrm{C}$, holding $2 \mathrm{~min}$. It was raised to $250^{\circ} \mathrm{C}$ and was held for $20 \mathrm{~min}$. Helium, as a carrier gas, was set to a column flow rate of $1 \mathrm{~mL} / \mathrm{min}$. Other instrumental parameters were set as: the electron energy was set at $70 \mathrm{eV}$, the ion source temperature set at $230^{\circ} \mathrm{C}$, the quadrupole temperature was $150^{\circ} \mathrm{C}$, the interface temperature was $280^{\circ} \mathrm{C}$ and the injector temperature set at $250^{\circ} \mathrm{C}$, the sample volume was $1 \mu \mathrm{L}$. Compounds were identified by matching their mass spectra with those of pure compounds whenever possible. Identification of structures/compounds of the peaks was supported by comparison to commercial mass spectral libraries in NIST (National Institute of Standards and Technologies) and Wiley format.

\section{Results and Discussion}

Yield of extractions.

The data in Table 1 show the results of factorial design with two factors of extraction time (three levels) and two solvents. Eighteen runs were carried out to cover all possible combination of two factors. Yield of extractions are expressed on the basis of amount of crude extracts. The results obviously show that, n-hexane was more effective solvent than methanol in extracting the ingredients of extracted oil of Pistacia Atlantica Kurdica nuts.

Table 1. Mass yield (\%) of extraction of Pistacia Atlantica Kurdica nuts using two extractor solvents.

\begin{tabular}{|c|c|c|c|c|c|c|c|c|}
\hline \multirow{4}{*}{$\begin{array}{l}\text { Extraction } \\
\text { Time } \\
\text { (hour) }\end{array}$} & \multicolumn{8}{|c|}{ Mass Yield (\%) } \\
\hline & \multicolumn{4}{|c|}{ n- hexane } & \multicolumn{4}{|c|}{ Methanol } \\
\hline & \multicolumn{3}{|c|}{ Replications } & \multirow{2}{*}{ Average } & \multicolumn{3}{|c|}{ Replications } & \multirow{2}{*}{ Average } \\
\hline & I & II & III & & I & II & III & \\
\hline 7 & 17.8 & 18.05 & 19.9 & 18.55 & 3.45 & 3.34 & 3.54 & 3.44 \\
\hline 11 & 34.2 & 32.98 & 33.6 & 33.59 & 3.49 & 3.98 & 4.09 & 3.85 \\
\hline 13 & 20.15 & 21.9 & 23.00 & 12.00 & 1.50 & 2.22 & 2.33 & 2.01 \\
\hline
\end{tabular}

It can be seen that the highest yield of extraction was achieved at 11 hours with $n$-hexane as extractor solvent. In this time, the percent of yield of extraction is $33.59 \%$, 
relative to weight of raw material of grounded nut which was placed in permeable thimble of extractor, while methanol only resulted in $3.85 \%$ mass yield. By increasing the extraction time from 7 to 11 hours the mass yield was increased and at 13 hours, the decrease was seen in extraction by $n$-hexane. This decrease maybe attributed to elimination of some extracted compounds present at low quantity in raw material and low boiling point. Also at time of 13 hours the decreasing of mass yield was seen for extraction with methanol. Thus we can conclude that extraction at high period of times leads to decreasing the extraction of component ingredients with low quantities. This result has been confirmed with GC-MS analysis.

The high polarity of methanol is main cause of low extraction with this solvent. In fact in solvent extraction, the polarity matching between solvent and extracted components is a very effective parameter. Given the structure properties and low polarity of extracted compounds, these results seem to be justifiable.

\section{Gas Chromatography - Mass Spectrometry Analysis}

MS is an analytical technique which identifies compounds based on the atomic sample composition of the molecules and their charge state [10]. Therefore, analysis of unknown samples is possible since MS does not require detailed prior knowledge of the sample composition. Although, MS library search will possibly lead to a correct identification if the spectrum of the unknown compound is really present in the library of instrument and the GC separation has been sufficiently efficient to obtain a clean mass spectrum.

In this study, many chemical compounds were extracted but some of themes successfully detected from the extracts with high qualities of match $(>\% 95)$ such as alpha-pinene, decane, dodecane, tetradecane, hexadecane, palmitic acid, oleic acid and linoleic acid ethyl ester. Although, many more compounds eluted from the chromatographic column, extensive searching of the mass spectral library tentatively identified many more components with lesser qualities of match.

Table 2 shows the extracted compounds in two times of extraction (11 and 13 hours) for fresh and dried samples. Also the resulted chromatograms in different conditions are showed in figure 1. These data's show that at time of 11 hours, many more compounds have been extracted related to 13 hours. It seems to be by increasing the extraction time, some of compounds with little quantities have been eliminated and peak areas of main compounds such as oleic acid and palmitic acid have increased. Also the drying of fresh samples not leads to elimination of compounds with high boiling point but other compounds have eliminated.

Alpha pinine was extracted from fresh and dry samples and seen in related chromatograms, thus the drying process is not leading to elimination of this compound. But, the quantity of extracted alpha-pinine is lesser than extracted from gum of Pistacia Atlantica Kurdica tree [11]. Figures 1, 2 and 3 show the chromatogram of extracted compounds with $n$-hexane as extractor solvent at 11 and 13 hours' time of extraction for fresh and dried samples. The notable result is high similarity between obtained chromatograms at different extraction times especially at 13 hours of extraction time for fresh and dried samples. This similarity maybe attributed to elimination the compounds with low boiling point at these conditions.

Table 2. The extracted compounds in two times of extraction (11 and 13 hours) for fresh and dried samples.

\begin{tabular}{|c|c|c|c|c|c|c|c|c|}
\hline \multicolumn{9}{|l|}{ Sample (extraction time) } \\
\hline Fresh (11 h) & & & Fresh (13 h) & & & Dried (13 h) & & \\
\hline Compound & R. T. ${ }^{\mathrm{a}}$ (min) & P. A.b (\%) & Compound & R.T. (min) & P. A. (\%) & Compound & $\begin{array}{l}\text { R. T. } \\
\text { (min) }\end{array}$ & $\begin{array}{l}\text { P. A. } \\
(\%)\end{array}$ \\
\hline ALPHA- PINENE & 5.65 & 0.67 & ALPHA- PINENE & 5.65 & 0.92 & ALPHA- PINENE & 5.65 & 0.67 \\
\hline Decane & 6.90 & 26.68 & Decane & 6.77 & 15.67 & Decane & 6.81 & 12.86 \\
\hline Undecane & 9.24 & 1.05 & Undecane & 9.23 & 0.25 & Undecane & 9.23 & 0.31 \\
\hline Dodecane & 9.99 & 9.25 & Dodecane & 9.90 & 4.87 & Dodecane & 9.92 & 4.16 \\
\hline 2-Tetradecene & 12.58 & 0.22 & N. E. ${ }^{c}$ & & & N.E. & & \\
\hline Tetradecane & 12.70 & 3.55 & Tetradecane & 12.67 & 1.64 & Tetradecane & 12.67 & 1.51 \\
\hline Hexadecane & 15.13 & 1.30 & Hexadecane & 15.12 & 0.55 & Hexadecane & 15.12 & 0.47 \\
\hline Hexadecanoic acid & 19.12 & 3.26 & Hexadecanoic Acid & 19.12 & 14.43 & Hexadecanoic acid & 19.21 & 12.32 \\
\hline 9-Octadecenoic acid & 20.80 & 4.38 & 9-Octadecanoic acid & 20.84 & 24.72 & 9-octadecanoic acid & 20.91 & 24.26 \\
\hline Linoleic acid ethyl ester & 20.88 & 0.58 & N.E. & & & N. E. & & \\
\hline Ethyl Oleate & 20.98 & 3.85 & Ethyl Oleate & 20.95 & 7.8 & N. E. & & \\
\hline
\end{tabular}

a. Retintion time, ${ }^{\mathrm{b}}$. Peak area, ${ }^{\mathrm{c}}$. Not extracted. 


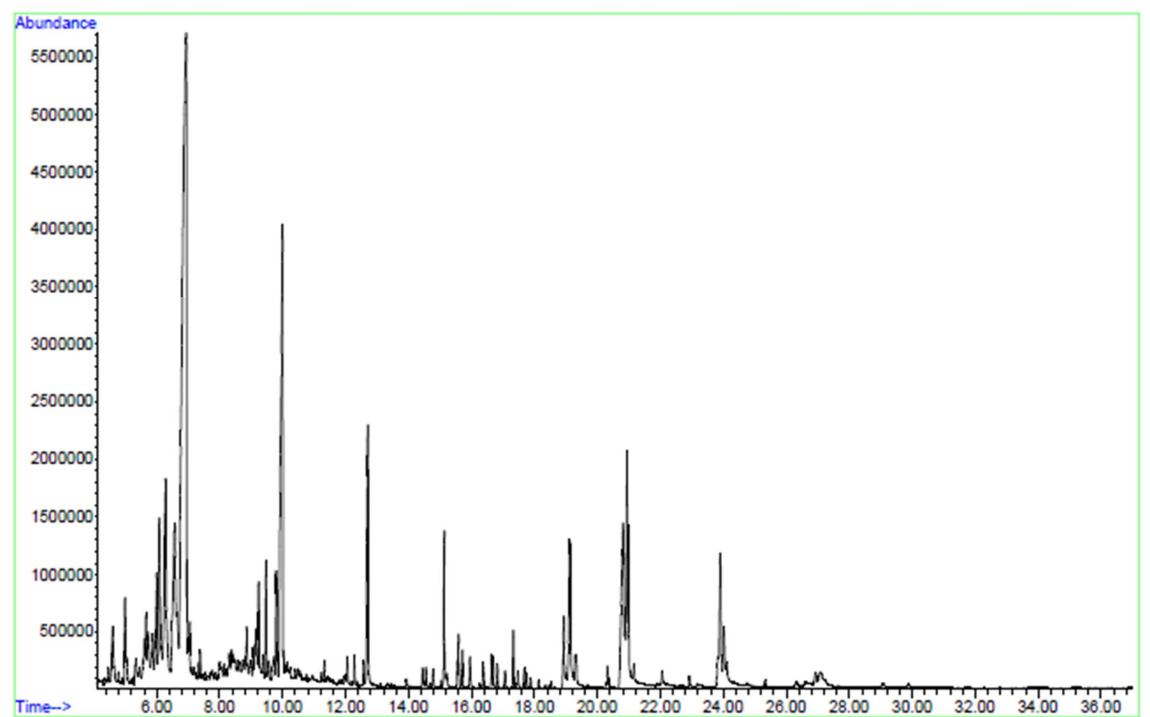

Fig. 1. The chromatogram of extracted compounds from fresh sample with n-hexane as extractor solvent at 11 hours' time of extraction.

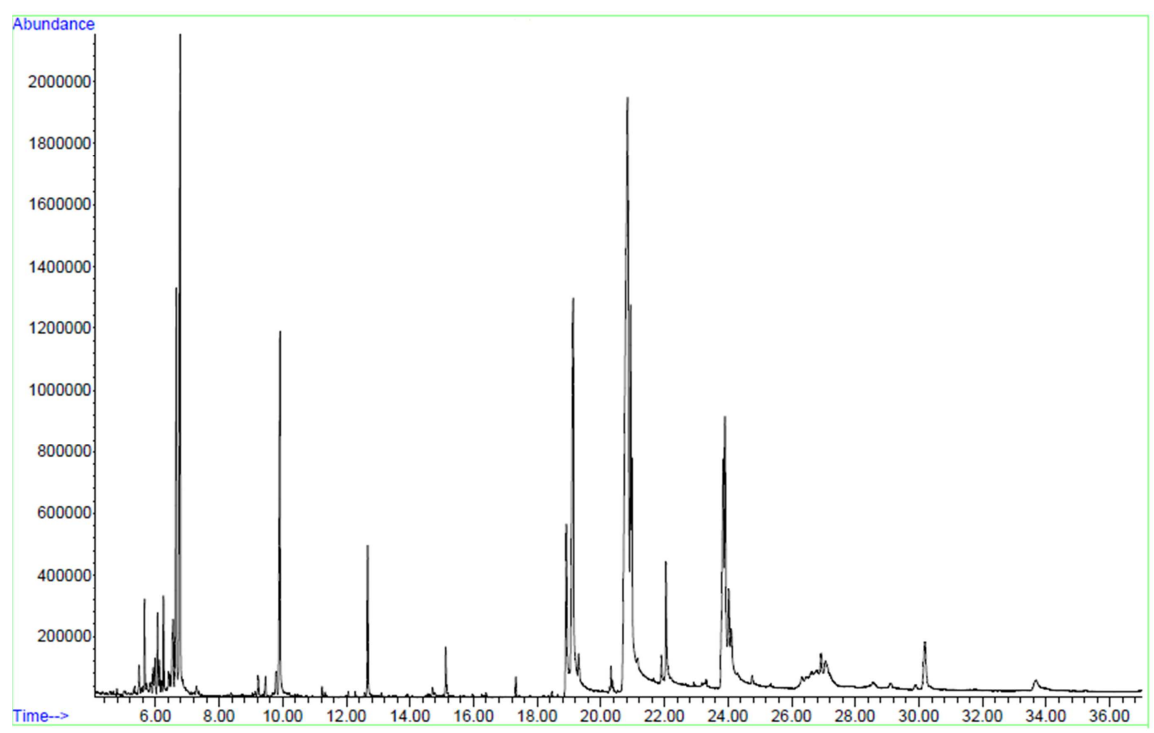

Fig. 2. The chromatogram of extracted compounds from fresh sample with n-hexane as extractor solvent at 13 hours' time of extraction.

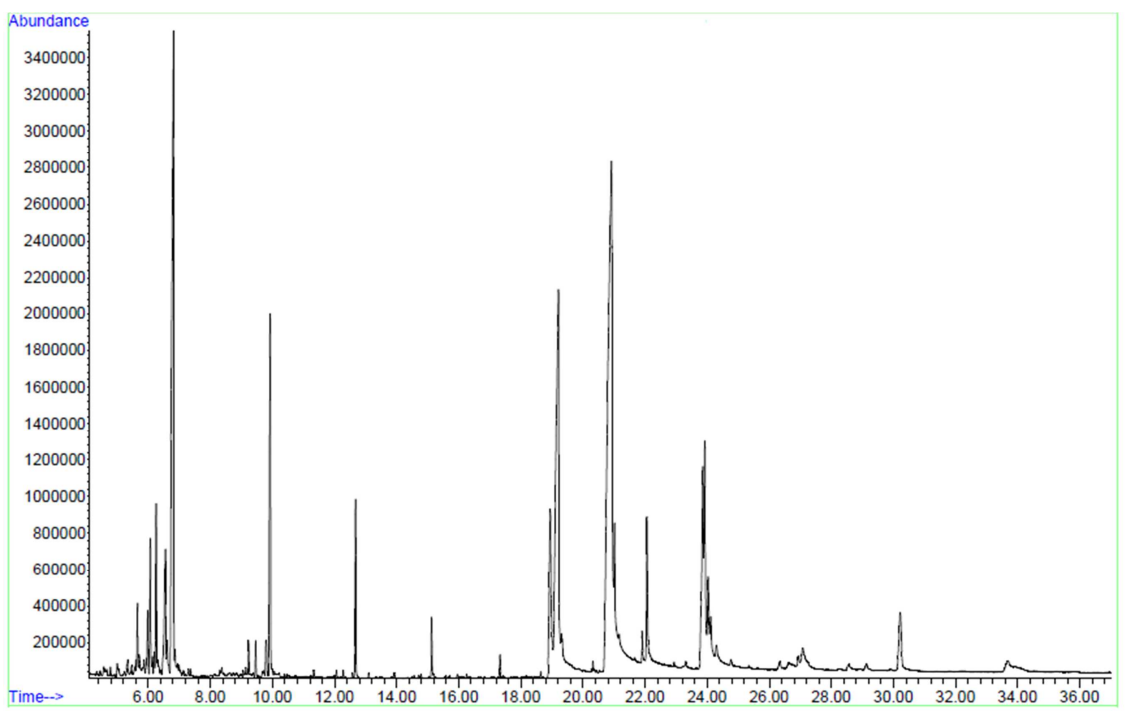

Fig. 3. The chromatogram of extracted compounds from dried sample with n-hexane as extractor solvent at 13 hours' time of extraction. 


\section{Design of Experiments}

The effect of extraction time and type of solvent were studied by implementation of general factorial design of experiment. To cover all possible combinations, eighteen extractions were required. Randomly, all runs were performed with three replicates.

The data yield of extraction was interpreted by Analysis of variance (ANOVA). The results are shown in Table 3. The effect of both factors (extraction time and type of solvent) was significant $(\mathrm{P}<0.0001)$. Furthermore, the interaction between two factors was significant $(\mathrm{P}<0.0001)$. Type of solvent is very effective factor on the yield of extraction. Indeed, polarity of solvent brings about high difference of extracted mass by $\mathrm{n}$-hexane and methanol. These results support considerable difference in extracted masses in various treatments. The 11 hour extraction time had the highest yield, whereas 7 hours extraction time had the lowest yield. The plot of the main effect for both factors is shown in Fig. 4, the behavior of each factor is shown individually.

Table 3. Results of analysis of Variance (ANOVA) for mass yield.

\begin{tabular}{llll|ll}
\hline Source & DF & SS & MS & F & P \\
\hline Type of solvent & 1 & 13.433 & 13.433 & 111.40 & 0.000 \\
$\begin{array}{l}\text { Extraction of time } \\
\text { Type of solvent }\end{array}$ & 2 & 11.495 & 5.748 & 47.66 & 0.000 \\
$\begin{array}{l}\text { Extraction of time } \\
\text { Error }\end{array}$ & 2 & 4.759 & 2.380 & 19.74 & 0.000 \\
Total & 12 & 1.447 & 0.120 & & \\
\hline
\end{tabular}

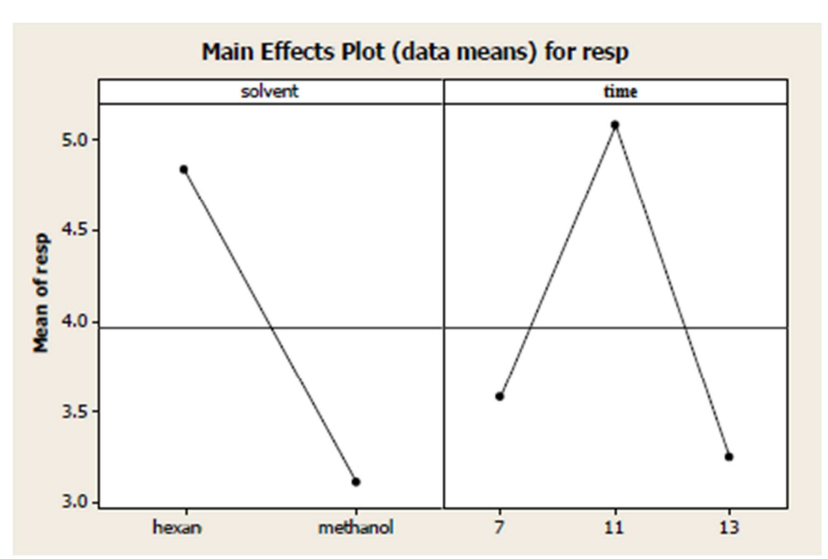

Fig. 4. Main Effects Plot (data means) for mass yield.

\section{Conclusions}

Soxhlet extraction has been the most widely-used extraction technique for several decades. Simplicity, low cost, specified consumption of the solvent and repetition of extraction cycles are some advantages of this method. The study shows that n-hexane extraction leads to higher yield than methanol extraction. The highest yield obtained with nhexane was $33.59 \%$; while the highest yield obtained by methanol was $3.85 \%$. The extracted oil was analyzed by GCMS and some fatty acids present in extracts taken from Pistacia Atlantica Kurdica nuts. The study shows that nhexane can be used to obtain maximum extracts because the polarity of compounds is low or medium in extracted oil. This study can be considered as the first report on the chemical compound of Pistacia Atlantica Kurdica nuts in Kurdistan lands (western Iran).

\section{References}

[1] Wheelwrigh E. G., "Medicinal Plants and their History", Dover Publications, New York (1974).

[2] Gottlieb O. R., Kaplan M. A., Borin M. R., "Biodiversidade um Enfoque Quimio-biológico" Editora UFRJ, Rio de Janeiro (1996).

[3] De Rougemont G. M., "A field guid to the crops of Britain and Europe", Collins (1989).

[4] Vasslios P., Papageorgiou A. N., Mellidis A. S., The Chemical Composition of the Essential Oil of Mastic Gum, J. Essent. Oil Res. 3(2): 107-110 (1991).

[5] Castola V., Bighelli A., Casanova J., Intraspecific Chemical Variability of the Essential Oil of Pistachia lentiscus L. from Corsica, Biochem. Syst. Ecol. 28(1): 79-88 (2000).

[6] Papageorgiou V. P., Sagredos A. N., Moser R., GLC-MS Computer Analysis of The Essential Oil of Mastic Gum, Chemica Chronika, New Series. 10: 119-124 (1981).

[7] Mgiatis P., Melliou E., Skaltsounis A. L., Chinou L. B., Mitaku. S., Chemical Composition and Antimicrobial Activity of the Essential Oils of Pistacia lentiscus var. chia, Planta Medica. 65(8): 749-752 (1999).

[8] Valérie C., Recent extraction techniques for solid matricessupercritical fluid extraction, pressurized fluid extraction and microwave-assisted extraction: their potential and pitfalls, Analyst, 126(7): 1182-1193 (2001).

[9] Maziar h., Study of herb diversity in the zagros forest (Case study: Kurdistan province), Int. J. Adv. Biol. Biom. Res. (IJABBR). 1(1): 25-34 (2013).

[10] De Hoffmann E., Stroobant V., "Mass Spectrometry: Principles and Applications", Wiley and Sons (2007).

[11] Sharifi M. S., GC-MS Analysis and Antimicrobial activity of the essential oil of the trunk exudates from Pistacia atlantica kurdica, J. Pharm. Sci. \& Res. 3(8): 1364-1367 (2011). 